\title{
Evaluating the Impact of Hippocampal Sparing During Whole Brain Radiotherapy on Neurocognitive Functions: A Preliminary Report of a Prospective Phase II Study
}

\author{
Shinn-Yn Lin ${ }^{1,2,3}$, Chi-Cheng Yang ${ }^{4}$, Yi-Ming Wu ${ }^{5}$, Chen-Kan Tseng ${ }^{1,2}$, Kuo-Chen Wei ${ }^{6}$, Yi-Chuan Chu \\ Hsiang-Yao Hsieh ${ }^{7}$, Tung-Ho Wu ${ }^{1,2}$, Ping-Ching Pai ${ }^{1,2}$, Peng-Wei Hsu ${ }^{6}$, Chi-Cheng Chuang ${ }^{6}$
}

Background: Whole brain radiotherapy (WBRT) is the treatment of choice for patients with brain metastases. However, neurocognitive functions (NCFs) decline due to impaired hippocampal neurogenesis might occur thereafter. It is hypothesized that conformal hippocampal avoidance during the course of WBRT (HA-WBRT) might provide meaningful NCF preservation. Our study aims to demonstrate the impact of delivering HA-WBRT on NCF changes in patients receiving WBRT.

Methods: Twenty-five patients who were referred for prophylactic cranial irradiation (PCI) or treating oligometastatic brain disease were enrolled in the study. Before the HA-WBRT course, all participants should receive baseline neurocognitive assessment, including memory, executive functions, and psychomotor speed. The primary endpoint was delayed recall, as determined by the change/decline in verbal memory [Wechsler Memory Scale $-3^{\text {rd }}$ edition (WMS III)- Word List score] from the baseline assessment to 4 months after the start of HA-WBRT.

Results: Only three patients belonged to the clinical setting of PCI; the remaining 22 patients had oligometastatic brain

\section{At a Glance Commentary}

Scientific background of the subject

Regarding the radiation-induced cognitive impacts, it is shown that impaired hippocampal neurogenesis might account for such neurocognitive impairment related to the delivery of whole brain radiotherapy. Therefore, it has been hypothesized that conformal hippocampal avoidance during the course of WBRT would provide meaningful preservation concerning neurocognitive functions.

\section{What this study adds to the field}

The incorporation of objective neurocognitive evaluations for brain metastatic patients has improved our understanding of neurocognitive functional outcomes in this population. It is also investigated whether neurocognitive functional preservation would be achieved via the integration of hippocampal sparing with the delivery of WBRT. disease. Regarding neurocognitive outcomes, no statistically significant differences were found between various NCF scores obtained at baseline and at post-radiotherapy intervals, in immediate verbal memory and non-verbal memory, except for delayed recall memory on Word List $(F=5.727, p=0.048)$.

Conclusions: Functional preservation by hippocampal sparing during WBRT could largely be achieved in this study, which also suggests that HA-WBRT should be a feasible technique preserving neurocognitive functions while maintaining intracranial control. (Biomed J 2015;38:439-449)

Key words: brain metastasis, hippocampus, hippocampus avoidance during whole brain radiotherapy, neurocognitive function, whole brain radiotherapy

S. Y. Lin and C. C. Yang have contributed equally to this article.

From the ${ }^{1}$ Department of Radiation Oncology, Chang Gung Memorial Hospital at Linkou, Chang Gung University College of Medicine, Taoyuan, Taiwan; ${ }^{2}$ Department of Medical Imaging and Radiological Sciences, College of Medicine, Chang Gung University, Taoyuan, Taiwan; ${ }^{3}$ Graduate Institute of Epidemiology and Preventive Medicine, College of Public Health, National Taiwan University, Taipei, Taiwan; ${ }^{4}$ Division of Clinical Psychology, Master of Behavioral Sciences, Department of Occupational Therapy, College of Medicine, Chang Gung University, Taoyuan, Taiwan; ${ }^{5}$ Department of Medical Imaging and Intervention, Chang Gung Memorial Hospital at Linkou, Chang Gung University College of Medicine, Taoyuan, Taiwan; ${ }^{6}$ Department of Neurosurgery, Chang Gung Memorial Hospital at Linkou, Chang Gung University College of Medicine, Taoyuan, Taiwan; ${ }^{7}$ Department of Neurology, Chang Gung Memorial Hospital at Linkou, Chang Gung University College of Medicine, Taoyuan, Taiwan

Received: Jan. 06, 2015; Accepted: May 12, 2015

Correspondence to: Dr. Chi-Cheng Chuang, Department of Neurosurgery, Chang Gung Memorial Hospital at Linkou. 5, Fusing St., Gueishan, Taoyuan, Taiwan (ROC) Tel.: 886-3-3281200 ext. 2412; Fax: 886-3-3285818; E-mail: ccc2915@cgmh.org.tw

DOI: $10.4103 / 2319-4170.157440$ 
$\mathrm{B}$ rain metastasis is a dismal diagnosis affecting approximately 200,000 Americans each year and up to $30 \%$ of patients with cancer. ${ }^{[1]}$ Generally, whole brain radiation (WBRT) with or without surgical resection has been the treatment of choice for patients with solitary brain metastasis and WBRT alone for those with multiple brain metastasis. Traditionally, WBRT has long been a practical and effective therapeutic modality for various settings of management in radiation oncology ${ }^{[2,3]}$ For example, the indications for WBRT include brain metastasis/metastases, the setting of prophylactic cranial irradiation (PCI) used for patients with limited-stage small cell lung cancer, and even some cases of extensive-stage small cell lung cancer. ${ }^{[4]}$ The rationale for WBRT is mainly based on the fact that it can target both microscopic and gross intracranial diseases.

In addition to providing rapid relief of neurologic symptoms and enhanced intracranial disease control, control of the metastatic brain lesion(s) by WBRT is generally the most important factor for stabilizing neurocognitive functions (NCFs). ${ }^{[5]}$ Paradoxically, NCF decline can also occur as a sequel of WBRT and is not negligible. Albeit complicated, the time course of WBRT-induced NCF decline can vary considerably according to the specific domains measured. Early decline occurs within the first 1-4 months after WBRT. ${ }^{[6]}$ The domains of radiation-related $\mathrm{NCF}$ decline mainly involve immediate and delayed verbal memory with/without non-verbal memory. ${ }^{[7,8]}$ For instance, Sun et al. reported that verbal memory function was most likely to deteriorate significantly after WBRT; by contrast, general cognitive functions and quality of life were not adversely influenced by WBRT. ${ }^{[7]}$

Over the past several decades, it has been understood that hippocampus plays an essential role in memory function. ${ }^{[9]}$ Also, not little evidence supports that impaired hippocampal neurogenesis caused by radiation-induced damage ${ }^{[10-13]}$ should be strongly associated with NCF impairment. ${ }^{[9,14]}$ Furthermore, several studies showed that isodose distribution in the hippocampus is closely related to NCFs in patients with primary brain tumors ${ }^{[15-17]}$ or in those with nasopharyngeal carcinoma. ${ }^{[14]}$ Consequently, it is hypothesized that conformal hippocampal avoidance during the course of WBRT (HA-WBRT) might lead to significant preservation in terms of cognitive function. ${ }^{[18-21]}$

This prospective cohort study aims to explore and evaluate the impact of the delivery of HA-WBRT on the extent of NCF changes in patients receiving prophylactic or therapeutic WBRT. As compared with previous related studies, it will also be investigated whether neurocognitive functional preservation would be achieved via the integration of hippocampal sparing with the course of WBRT.

\section{METHODS}

\section{Study patients, eligibility, and clinical setting}

Patients with primary lung cancer referred for PCI or adults with pathologically confirmed non-hematopoietic malignancy and brain metastasis who had fair to good performance status represented by Karnofsky Performance Status (KPS) score 70 or Eastern Cooperative Oncology Group ECOG) score 2 were eligible for the study. Also, the number and extent of brain metastatic lesions should be no more than three metastatic foci, with the greatest diameter being less than $4 \mathrm{~cm}$. Of note, this inclusion criterion was confirmed in patients by performing brain magnetic resonance imaging (MRI) after they underwent craniotomy with tumor removal and befo re the course of WBRT if potentially eligible patients were surgically resected cases. Thus, in addition to the patients referred for PCI, most of our enrolled patients had to correspond with the definition of oligometastatic brain disease, implying that the number of metastatic foci was three or less shown on brain MRI. ${ }^{[22,23]}$ Accordingly, patients must fall into RTOG recursive partitioning analysis (RPA) class I or II. ${ }^{[24,25]}$ Using the above inclusion criteria, new treatment techniques can hopefully be tested on homogeneous patient groups of these classes.

Given the concerns of the safety issue arising on reducing the dose delivered to hippocampal areas below the therapeutic level as it might potentially increase the risk of hippocampal metastasis, patients with MRI-identified metastasis within $5 \mathrm{~mm}$ perihippocampally should be excluded. Other exclusion criteria were clinical suspicion of leptomeningeal spreading, history of prior radiotherapy (RT) delivered to brain/head region for any reason, and contraindication for receiving contrast-enhanced MRI examination.

Between March 2013 and the end of April 2014, totally 25 patients were enrolled in the current prospective study. Based on our predefined inclusion eligibility, the recruited patients fell into two main groups. Moreover, the study protocol had been approved by the institutional review board (IRB) at our institute (IRB 103-1090C and 101-4151B) and written informed consent was obtained from each enrolled and eligible patient or the person authorized to give consent. Importantly, our research is a principle investigator (PI)-initiated study; neurosurgeons and radiation oncologists constitute the main investigators taking responsibility for recruiting appropriate patients for this prospective study.

\section{Pretreatment evaluations/assessments}

No matter whether the enrolled patients were surgically resected cases or not, eligible patients without detected brain metastasis within a 5-mm margin around either hip- 
pocampus on gadolinium-enhanced MRI should be obtained within 1 month prior to the planned course of HA-WBRT. Similarly, relevant physical examination and staging oncological surveys should be performed within 30 days before the initiation of HA-WBRT course. Most importantly, all participants must receive the baseline neurocognitive assessment (mentioned below) which is planned to be administered within 2 weeks before the start of HA-WBRT course. Besides, the time interval between brain MRI examination and baseline neurocognitive testing should be as short as possible, preferably within 2 weeks.

\section{Neurocognitive assessments}

A selective neurobehavioral test battery is administered [Table 1]. The neurobehavioral measures mainly evaluate several domains assumed to be sensitive to the tumor involvement and cranial RT; all tests were administered by a trained clinical research associate under the supervision of a neuropsychologist.

Since the delivery of HA-WBRT instead of conventional WBRT aims to diminish the extent of neurotoxic impact on the hippocampus, which is significantly associated with memory functions, ${ }^{[9,15,18,21,26]}$ three main aspects of NCF, including memory functions, executive functions, and psychomotor speed, were evaluated. First, the selected subtests of the Wechsler Memory Scale $-3^{\text {rd }}$ edition (WMS-III) were used to evaluate patients' verbal and non-verbal episodic memory. ${ }^{[27]}$ Second, the Modified Card Sorting Test was used to assess both conceptual formation and mental shifting, ${ }^{[28]}$ which have been documented to be the major components of executive functions. Besides, the Digit Span (DS) subtest of the Wechsler Adult Intelligence Scale $-3^{\text {rd }}$ edition (WAIS-III ${ }^{\circledR}$ ) was used to examine the verbal working memory. ${ }^{[29]}$ Finally, in order to determine patients' performance on the psychomotor speed, Psychomotor Speed Index (PSI), which is derived from the composite score of Digit Symbol Coding Subtest (DSS) and Symbol Searching Subtest (SS) of the WAIS-III ${ }^{\circledR}$, was used.

\section{Radiation therapy treatment planning and delivery}

All patients had to undergo a computed tomography (CT) simulation scan encompassing the entire head region with $1.25-\mathrm{mm}$ slice thickness using a thermoplastic mask for immobilization. Bilateral hippocampi were contoured on T1-weighted sequence of axial MRI with gadolinium contrast enhancement. Contouring was initiated at the most caudal portion of the crescent-shaped floor of the temporal horns and continued posterio-cranially along the medial edges of temporal horns [Figure 1A and B]. The hippocampal contouring for all patients in our study was confirmed by the same neuroradiologist; appropriate anatomical contouring was also modified and verified by using T1-weighted MRI coronal and sagittal sequences. Furthermore, in order to allow sharp dose fall-off between the hippocampal areas and the planning target volume (PTV) for the whole brain, the hippocampal avoidance zone (HA zone) was generated by expanding the hippocampal outlines with a margin of $5 \mathrm{~mm}$ volumetrically. Accordingly, the whole-brain PTV was defined as whole-brain parenchyma excluding the HA zone. ${ }^{[30]}$

To achieve conformal hippocampal sparing during the delivery of WBRT, the technique of volumetric modulated arc therapy (VMAT) was employed with using Linac-based RapidArc ${ }^{\circledR}$. For inverse planning optimization, Eclipse v. 8.6 software was used with the analytical anisotropic algo-

Table 1: A selective neurocognitive test battery

\begin{tabular}{|c|c|c|}
\hline Neurocognitive tests & Domain & Description \\
\hline \multicolumn{3}{|l|}{ WAIS-III } \\
\hline Digit span & Working memory & Related to prefrontal cortex \\
\hline Digit symbol substitution & Psychomotor speed & $\begin{array}{l}\text { Related to the function of white matter tract (a measure } \\
\text { of psychomotor speed and sustained attention) }\end{array}$ \\
\hline Symbol Searching & Psychomotor speed & $\begin{array}{l}\text { Related to white matter tract (to measure the speed of } \\
\text { visual-perceptual discrimination and scanning) }\end{array}$ \\
\hline Verbal learning & Verbal memory & \\
\hline \multicolumn{3}{|l|}{ WMS-III } \\
\hline Word lists (I) & Immediate memory & \multirow{2}{*}{$\begin{array}{l}\text { To evaluate auditory memory of verbal information } \\
\text { that is without context }\end{array}$} \\
\hline Word lists (II) & Delayed memory & \\
\hline Non-verbal learning & Visual memory & \\
\hline \multicolumn{3}{|l|}{ WMS-III } \\
\hline Visual reproduction (I) & Immediate memory & \multirow{2}{*}{$\begin{array}{l}\text { To measure the ability to remember visually presented } \\
\text { information }\end{array}$} \\
\hline Visual reproduction (II) & Delayed memory & \\
\hline \multicolumn{3}{|l|}{ Executive function } \\
\hline MCST & Executive function & Related to conceptual formation and mental shifting \\
\hline
\end{tabular}




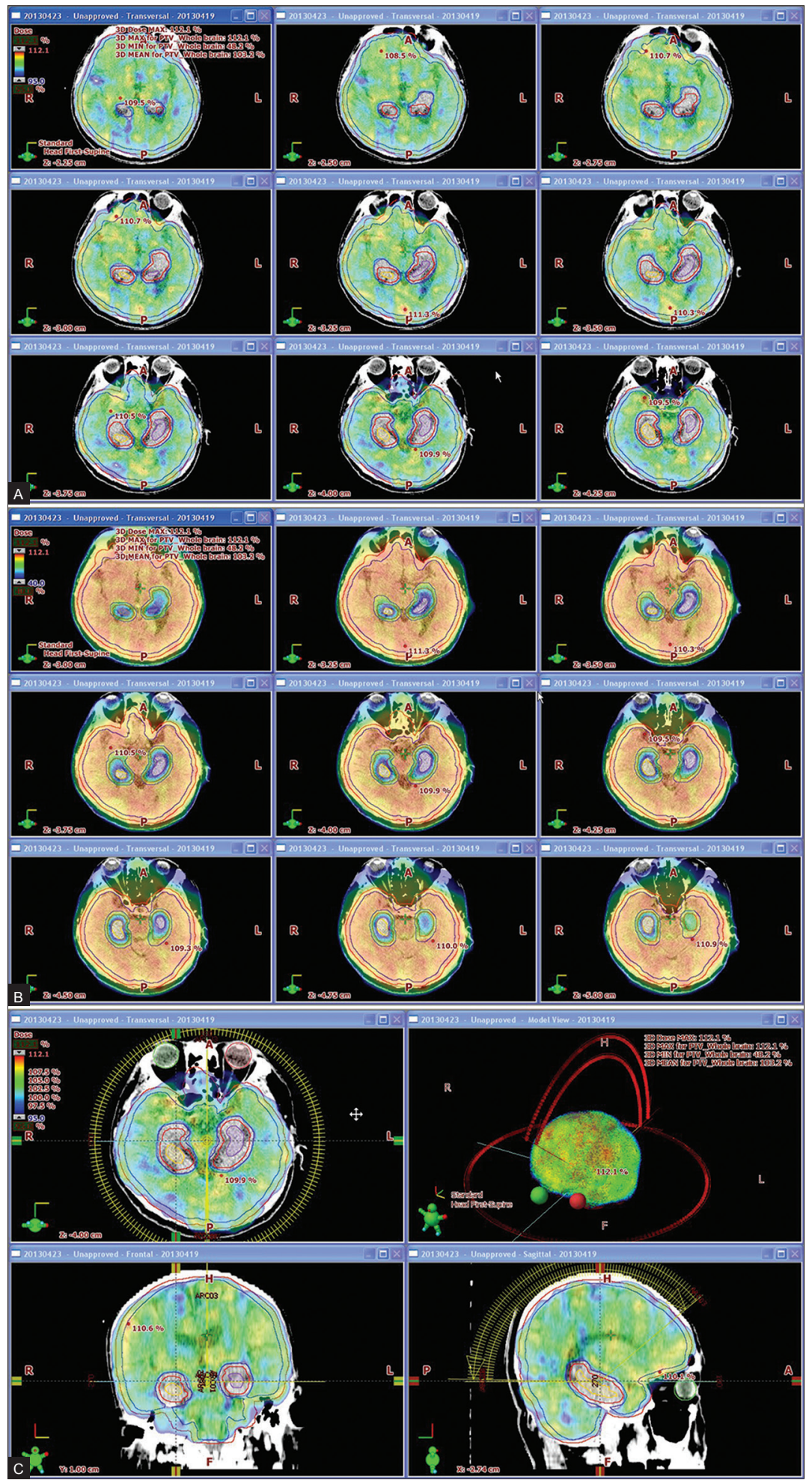

Figure 1: Axial isodose distribution and volume modulated arc therapy (VMAT)-based treatment planning of one representative patient at the level of bilateral hippocampal areas for hippocampal sparing during the delivery of WBRT. (A) The hippocampal avoidance zone (red) created by expanding the hippocampal contouring (orange at right and purple at left) with a 5-mm volumetric margin to compensate for setup uncertainty and sharp dose falloff. Appropriate anatomical contouring was also checked and verified using T1-weighted MRI coronal and sagittal series by the same neuroradiologist. (B) The isodense region without added color display representing the hippocampal contouring. The $40 \%$ isodose surface indicates what our VMAT treatment plan has attempted to achieve, the so-called hippocampal sparing. (C) In addition to the isodose distribution shown on representative axial, coronal, and sagittal planes, the right upper panel illustrates the arrangement of VMAT treatment planning by virtue of four arcs, in which two non-coplanar arcs were designed. 
rithm (AAA) and all treatment plans were delivered by using Linac Varian-iX. Import antly, our preliminary planning study conducted during the past several years has shown that the utilization of VMAT technique via Lin ac-based RapidArc to achieve conformal avoidance of hippocampal areas has become mature and optimized (unpublished results). Briefly, four arcs of VMAT in way of non-coplanar arrangement were designed [Figure 1C]. In terms of dose prescription, a dose of $30 \mathrm{~Gy}$ in 12 fractions was prescribed to whole-brain PTV if the role of RT was considered therapeutic for treating oligometastatic brain disease or adjuvant following craniotomy with tumor removal.

\section{Imaging and clinical follow-up}

Identical to pre-RT assessment via brain MRI and a battery of neurocognitive assessment for the enrolled patients, imaging and clinical follow-up were arranged for the study participants and performed at pre-specified post-RT intervals, which are 4 months and 12 months after the start of HA-WBRT course, respectively.

Although follow-up duration is too short for the common oncological outcomes to occur, the subjects were kept under close observation in order to detect any intracranial failures by using brain MRI performed at 4 months after the start of cranial RT. Basically, three patterns of central nervous system (CNS) failure were found: local failure, distant brain parenchymal failure, and development of leptomeningeal disease. ${ }^{[31]}$ Also, for each case with brain parenchymal failure, the site (s) of CNS failure shown on follow-up MRI was co-registered, fused, and mapped with the corresponding previous isodose distribution in CT treatment planning to assure whether the site (s) of such brain parenchymal failure would fall out of the zone of hippocampal sparing-related underdose regions.

\section{Statistical considerations and analyses}

Our primary endpoint was delayed recall, as determined by the decline/change in either verbal memory (WMS III - Word Lists delayed recall score) or non-verbal memory [WMS III - Visual Reproduction (VR) delayed recall score] from baseline assessment to 4 months after the start of HA-WBRT. Consequently, in order to compare patients' NCFs, which included memory, executive functions, and psychomotor speed, before and after the HA-WBRT course, the repeated measure analysis of variance (ANOVA) was used. The statistical significance of our analyses was set as a $p$ value of less than 0.05 after the Bonferroni adjustment. The commercially available software (SPSS Version 20.0) was employed.

Besides, regarding the intracranial progression evaluated via brain MRI, the Kaplan-Meier estimator was used to compute the median time to radiographic progression for our studied patients (along with 95\% confidence intervals). The Cox proportional hazards regression model was employed to investigate the effects of histology, RPA class, and other available covariates on time to radiographic progression.

\section{RESULTS}

\section{Study patients, eligibility, and clinical setting}

By the end of April 2014, totally 25 patients had been enrolled in the current prospective study for investigating the feasibility and impact of hippocampal sparing during WBRT course on the change or preservation of neurocognition. Based on our predefined patient eligibility, there were two main groups of recruited patients. Actually, there were only three patients belonging to the standard setting of what is called PCI, including two patients with limited-stage small cell lung cancer who had achieved good clinical response to primary chemoradiation and one case with locoregionally advanced non-small cell lung cancer that had also shown significant objective response to primary concurrent chemoradiation. In addition to the three patients falling into the clinical setting of conventional PCI, the remaining 22 patients had solitary brain metastasis or oligometastatic brain disease. Noticeably, the majority of patients were referred from the Department of Neurosurgery after they had undergone craniotomy with removal of the main metastatic tumor, including 15 cases with a single brain metastasis and 4 patients with oligometastatic brain disease.

\section{Patient demographics and clinical characteristics}

In this preliminary report, as shown in Table 2, the patients enrolled in the study initially included 15 males and 10 females. The median age at registration was 57.6 years. All eligible patients had a fair performance status, as indicated by a KPS of 70 or more; approximately one-third of the patients had even a good KPS (90). Except for the seven Patients whose primary cancer arose from miscellaneous malignancies, most of the patients had lung cancer and breast cancer as the primary cancer. Regarding the number of brain metastatic foci, more than two-thirds of the patients had only one solitary brain metastasis and even zero MRI-detected lesions, implying the clinical situation of PCI.

Furthermore, 19 out of 25 patients $(76 \%)$ had undergone craniotomy with gross tumor removal for the space-occupying lesion causing active symptoms, and these included 15 cases with a single brain metastasis, 3 patients with two brain metastatic lesions, and 1 case with three metastatic foci at diagnosis of intracranial dissemination. Therefore, concerning the role of WBRT, it was therapeutic for oligometastatic brain disease for 7 patients (28\%); by contrast, 
Table 2: Summary of patient demographics, tumor and disease characteristics

\begin{tabular}{|c|c|c|}
\hline Characteristics & $\begin{array}{c}\text { No. of } \\
\text { patients (\%) }\end{array}$ & $\begin{array}{c}\text { No. of } \\
\text { lesions (\%) }\end{array}$ \\
\hline Number of patients & 25 & \\
\hline Number of brain metastatic lesions & & 29 \\
\hline \multicolumn{3}{|l|}{ Age at registration, years } \\
\hline Median & 57.6 & \\
\hline Mean (range) & $\begin{array}{c}58.9(28.3- \\
79.1)\end{array}$ & \\
\hline \multicolumn{3}{|l|}{ Gender } \\
\hline Male & $15(60 \%)$ & \\
\hline Female & $10(40 \%)$ & \\
\hline \multicolumn{3}{|l|}{ Performance status before the course of brain RT } \\
\hline KPS 90 & $8(32 \%)$ & \\
\hline $70 \mathrm{KPS}<90$ & $17(68 \%)$ & \\
\hline \multicolumn{3}{|l|}{ Performance status (ECOG) } \\
\hline $0-1$ & $19(76 \%)$ & \\
\hline 2 & $6(24 \%)$ & \\
\hline \multicolumn{3}{|l|}{ Histological subtype of primary cancer } \\
\hline Lung, NSCLC & $12(48 \%)$ & \\
\hline Lung, SCLC & $3(12 \%)$ & \\
\hline Breast & $3(12 \%)$ & \\
\hline Others*/Unknown $\dagger$ & $6 / 1(28 \%)$ & \\
\hline Number of brain metastatic lesions at diagnosis & 29 & \\
\hline $0, \mathrm{PCI}$ & $3(12 \%)$ & \\
\hline 1 & $17(68 \%)$ & \\
\hline 2 & $3(12 \%)$ & \\
\hline 3 & $2(8 \%)$ & \\
\hline Number of surgical cavities & & 19 \\
\hline \multicolumn{3}{|l|}{ Extent of resection } \\
\hline En-bloc gross total & & $17(89.5 \%)$ \\
\hline Subtotal & & $2(10.5 \%)$ \\
\hline \multicolumn{3}{|l|}{ Role of WBRT } \\
\hline PCI & $3(12 \%)$ & \\
\hline Adjuvant post-craniotomy & $15(60 \%)$ & $15(51.7 \%)$ \\
\hline Therapeutic for oligometastatic brain disease & $7(28 \%)$ & $14(48.3 \%)$ \\
\hline \multicolumn{3}{|l|}{ Status of primary cancer } \\
\hline Stable/controlled & $15(60 \%)$ & \\
\hline Uncontrolled/progressive & $10(40 \%)$ & \\
\hline \multicolumn{3}{|l|}{ Status of extracranial metastasis } \\
\hline Stable/controlled & $14(56 \%)$ & \\
\hline Uncontrolled/progressive & $11(44 \%)$ & \\
\hline \multicolumn{3}{|l|}{ RTOG RPA class $\S$} \\
\hline Class I & $10(40 \%)$ & \\
\hline Class II & $15(60 \%)$ & \\
\hline
\end{tabular}

*: One case with adenocarcinoma from unknown primary, one case with hepatocellular carcinoma, one case with esophageal cancer, one case with neuroendocrine carcinoma of uterine cervix, one case with malignant melanoma, and one case with liver angiosarcoma; $\dagger$ : One enrolled patient had brain metastatic adenocarcinoma whose primary cancer was not found; $\$$ : According to our predefined criteria, oligometastatic brain disease indicates that the number of brain metastatic lesions is three or less; §: Based on recursive partitioning analyses (RPA) performed by Radiation Therapy Oncology Group (RTOG), three classes were suggested: Class I: patients with KPS greater than or equal to $70,<65$ years of age with controlled primary and no extracranial metastases; Class III: KPS <70; Class II: all others it was an adjuvant following craniotomy in 15 cases $(60 \%)$ for whom en-bloc gross tumor resection had already been accomplished. Since there were no grossly residual lesions left among these 15 patients who had solitary brain metastasis and had undergone gross tumor removal, actually 18 out of 25 patients (72\%) had no grossly residual brain lesion(s) during registration of this study, including 3 PCI patients and 15 surgically resected cases.

According to RTOG RPA classification, 15 out of 25 patients $(60 \%)$ belonged to class II and the remaining 10 patients $(40 \%)$ were considered the most favorable group, i.e. class I. In addition, among the 15 patients who had either uncontrolled primary cancer or progressive status of extracranial metastasis, there were 6 patients experiencing both progressive primary cancer and uncontrolled extracranial metastasis.

\section{Neurocognitive outcomes}

In our earlier report, compliance with NCF testing was $100 \%$ at baseline and $57 \%$ at 4 months after the initiation of HA-WBRT course. The majority of non-compliance might be attributed to patient-related factors, mainly a deteriorated performance status. The chart in Figure 2 summarizes the trial flow of this preliminary report.

As illustrated in Figure 3A, regarding the verbal memory, no significant difference was found in the scaled scores of short-term memory on the Word List (WL) $(F=0.368$, $p=0.563$ ) between before and after the course of HA-WBRT, whereas a significant difference was found in the long-term memory on the WL $(F=5.727, p=0.048)$ between these two evaluations. As for the non-verbal memory, no significant difference was noted in any scaled score on the VR between pre- and post-HA-WBRT course.

With respect to the executive functions [Figure 3B], when comparing the performances before the HA-WBRT course and 4 months after the start of HA-WBRT course, we found no statistically significant difference on the DS $(F=0.000, p=1.000)$ and the Modified Card Sorting Test [for Completed Categories (CC): $F=0.149, p=0.711$; for Preservative Errors (PE): $F=1.211, p=0.307]$.

Concerning the performances of psychomotor speed [Figure 3C], a similar finding was noted in accordance with the above NCF results. The performances on the DSS $(F=0.030, p=0.867)$ and the SS $(F=0.578$, $p=0.472)$ were not significantly different between before and after the HA-WBRT course.

\section{Intracranial control and early oncological outcomes}

Despite insufficient follow-up, up to now, as shown in Table 3, no any intracranial failure has occurred in the 


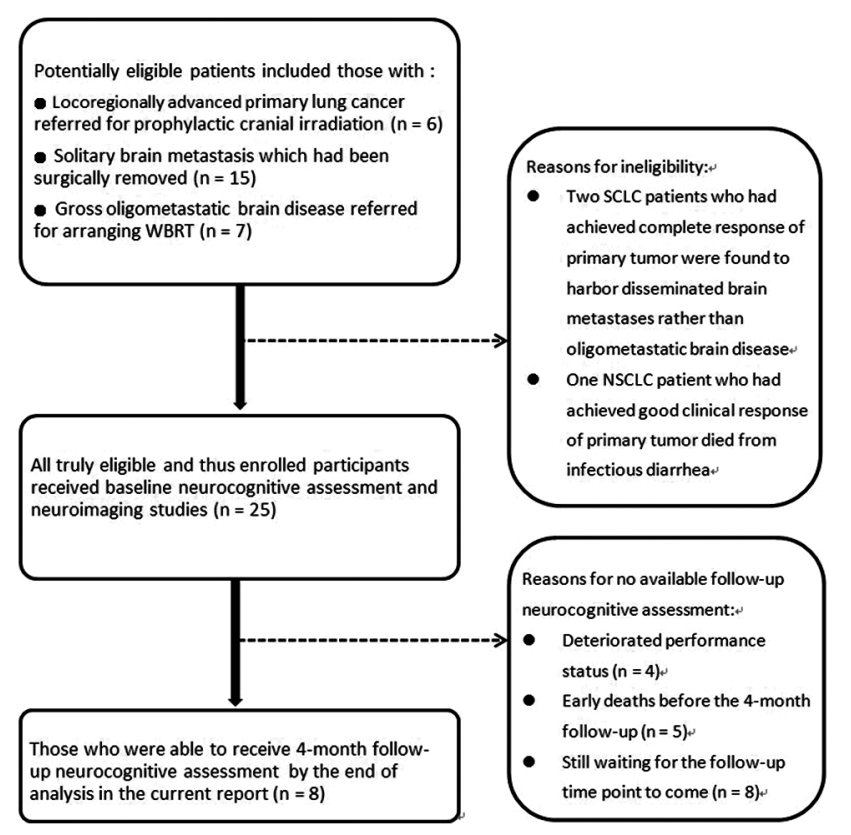

Figure 2: The chart diagram of this preliminary report. underdose region, indicating that the zone of hippocampal sparing might be safely free from CNS failure and that all brain metastatic foci have indeed been irradiated appropriately with our predefined prescribed dose (i.e. $3000 \mathrm{cGy}$ in 12 fractions). Furthermore, there were four patients experiencing treatment failure within the $\mathrm{CNS}$, including two cases developing leptomeningeal disease and two patients experiencing local failure. Besides, one patient experiencing intracranial local failure also developed distant brain parenchymal failure metachronously.

By the end of April 2014, post-RT MRI follow-up was feasible in 10 patients at 4 months after the start of HA-WBRT course, among whom CNS tumor control was generally quite good. One patient suffered early CNS failure both locally and distantly, two patients still had a residual lesion which had not been managed surgically or radiosurgically, and the remaining seven patients still remained free of any CNS failure at this time point.

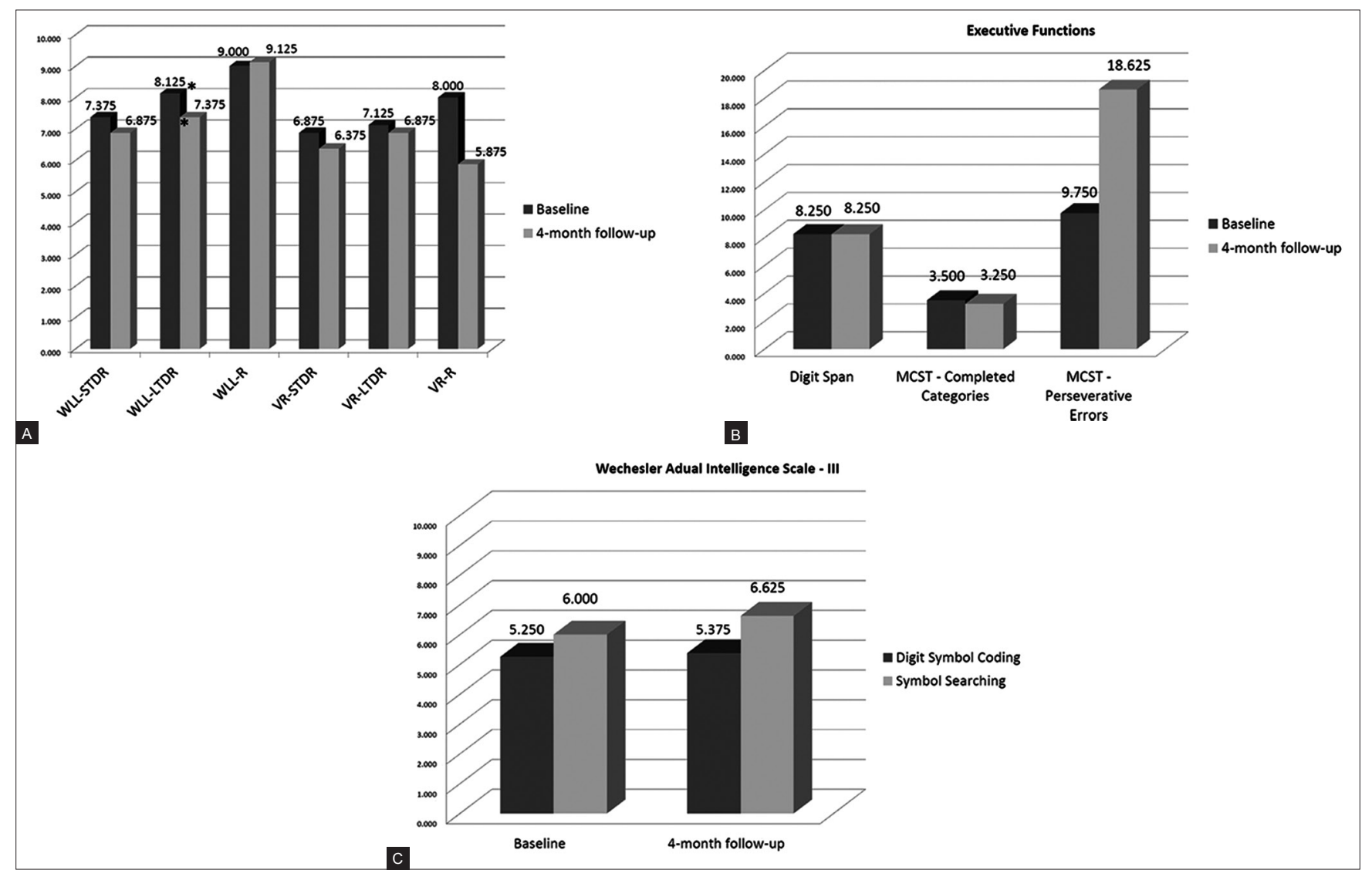

Figure 3: Neurocognitive performance scores regarding three major domains, memory, executive functions, and psychomotor speed, respectively. (A) Performances on the Wechsler Memory Scale - 3rd version at baseline and 4-month follow-up evaluations. (B) Performances on the Modified Card Sorting Test at baseline and 4-month follow-up evaluations. (C) Performances on the Psychomotor Speed Index of the Wechsler Adult Intelligence Test $-3^{\text {rd }}$ version at baseline and 4-month follow-up evaluations. The presence of asterisk $(*)$ indicates the specific neurocognitive test which was demonstrated to be statistically significant between the baseline score and the one obtained at 4-month follow-up assessment. 
Table 3: Early oncological outcomes and intracranial control

\begin{tabular}{|c|c|c|}
\hline $\begin{array}{l}25 \text { studied patients harboring } 29 \text { MRI- } \\
\text { detected metastatic foci at enrollment }\end{array}$ & $\begin{array}{l}\text { No. of } \\
\text { patients }\end{array}$ & $\begin{array}{l}\text { No. of } \\
\text { lesions }\end{array}$ \\
\hline \multicolumn{3}{|l|}{ Patterns of CNS failure/control } \\
\hline Local failure & $2 *$ & 2 \\
\hline Distant brain parenchymal failure & $1^{*}$ & 1 \\
\hline Leptomeningeal disease $^{\dagger}$ & 2 & \\
\hline †HA-related relapse & None & \\
\hline Available brain MRI at 4 months & 10 patients & \\
\hline NER & 7 & \\
\hline Residual lesion still present & 2 & 2 \\
\hline Early CNS failure & $1^{*}$ & \\
\hline \multicolumn{3}{|l|}{ Premature withdrawal from this study } \\
\hline Suicide/accidents & 1 & \\
\hline Personal reasons & 1 & \\
\hline Status of survival and causes of death & 25 patients & \\
\hline Mortality & 5 & \\
\hline Cancer-specific death & 4 & \\
\hline Non-neurologic death & 2 & \\
\hline Neurologic death & 2 & \\
\hline Alive with CNS failure & 2 & \\
\hline Alive with active extracranial disease & 1 & \\
\hline $\begin{array}{l}\text { Alive, progression-free or receiving } \\
\text { active cancer treatment }\end{array}$ & 17 & \\
\hline
\end{tabular}

*: There was one patient experiencing early CNS failure along the original surgical resection cavity less than 4 months after receiving HA-WBRT; This patient was later found to have another new brain metastatic lesion indicating distant brain parenchymal failure; ’’: Leptomeningeal disease: A conservative definition of leptomeningeal disease was used, including obvious leptomeningeal enhancement on MRI involving the brain (in the sulci of cerebral hemispheres, the subependyma, the cranial nerves, of folia of the cerebellum), spinal cord, or cauda equina; : HA indicates hippocampal avoidance in this study. In order to make sure whether the site (s) of brain parenchymal failure would unfortunately fall within the zone of hippocampal sparing-related underdose region, the site (s) of CNS failure shown on follow-up MRI would be fused with the isodose distribution in previous CT treatment planning of HA-WBRT

\section{Feasibility and significance of this prospective study (HA-WBRT)}

Since no any intracranial failure was associated with the underdose area represented by the zone of hippocampal avoidance, we believe that reducing the dose delivered to the hippocampal areas when treating patients with WBRT might not compromise intracranial tumor control at all. Furthermore, several related studies have consistently supported selectively reducing the dose delivered to the hippocampus when treating oligometastatic patients with WBRT. ${ }^{[22,23,32,33]}$

Although the follow-up duration was too short for us to perform analytic statistical testing with regard to intracranial disease control and survival outcomes, our preliminary results clearly showed that specific NCFs (i.e. verbal and non-verbal learning memory, executive functions, and psychomotor speed) did not significantly change or decline in patients who had undergone hippocampal sparing during the WBRT course.

\section{Strengths of the current study}

To the best of our knowledge, there has been little evidence concerning the neurocognitive outcomes for non-primary brain tumor patients, let alone cancer patients with brain metastasis in the Taiwanese population. Therefore, this prospective clinical study with neurocognitive outcome research might be a pioneering study focusing on neurocognitive outcomes in the field of neuro-oncology in an eastern Asian country. By virtue of this prospective Phase II study carried out in Taiwan, we were able to obtain preliminary results, which will guide us when formulating and designing the future study protocols. Moreover, all contouring tailored to the imaging anatomy of bilateral hippocampal areas was delineated and verified by the same neuroimaging radiologist, rather than neurosurgeons or radiation oncologists only. Similarly, all neurocognitive assessments were administered under the supervision of a well-experienced neuropsychologist, who had selected a neurobehavioral test battery specifically for the current study.

\section{Neurocognitive status/change before and after HA-WBRT}

Multi-domains of NCF were examined in this study, and our results consequently showed no significant differences in memory, executive functions, and psychomotor speed between baseline and 4 months after the start of HA-WBRT course, except for the long-term delayed recall of verbal memory. In fact, these support the finding that conformal sparing of hippocampal areas during the delivery of WBRT might preserve patients' NCF mostly or alleviate the extent of NCF changes ${ }^{[23,34]}$ Indeed, it has been documented that WBRT is associated with late neurotoxicity resulting from brain irradiation and will induce multi-faceted difficulties in patients including memory, attention, and motor control; $;[8,35]$ therefore, HA-WBRT was developed to preserve cortical NCFs even after cranial RT. ${ }^{[20,23,36]}$ Although quite a few researches ${ }^{[23,34,37,38]}$ have conceptually hypothesized that HA-WBRT might mitigate the cognitive decline after brain RT, studies concerning the dynamic changes of NCF before and after HA-WBRT are still limited and preliminary. ${ }^{[39]}$ For example, Gondi et al. have conducted a Phase II clinical trial, RTOG 0933, to investigate the effects of HA-WBRT in patients with brain metastases and they evaluated memory performances by using the Hopkins Verbal Learning Test (HVLT) at 4 months after initiating brain RT. ${ }^{[18,21]}$ Our prospective study further provides the preliminary evidence that specific NCFs in patients with oligometastatic brain disease are quite stable between baseline assessment and those evaluated at 4 months after HA-WBRT. 


\section{Potential limitations of the present study}

Although this prospective study might be limited by the fact that there was no real control group for which conventional WBRT without hippocampal sparing was delivered, actually each patient indeed serves as his/her own control because the difference in scores obtained at baseline and at pre-specified post-treatment intervals was calculated. Furthermore, there are other potential patient-related, disease-related, and treatment factors which also play a role in affecting patients' NCF change, such as the extent of brain edema caused by the metastatic brain focus per se or surgical intervention, general performance status, the confounding effect of increased intracranial pressure (IICP), nutritional condition, and electrolyte imbalance. Nevertheless, our predefined strict inclusion criteria have focused our enrolled patients on those with satisfactory performance status and a limited number and burden of metastatic brain foci. Therefore, it is assumed that the impact of potential confounders can be kept as negligible as possible provided that the enrolled patients really meet the eligibility criteria. Last but not least, the patient population in the current study seems quite heterogeneous because we enrolled two main patient subgroups; one subgroup represented the patients who were referred for PCI and the other represented those with oligometastatic brain disease. Also, patients with oligometastatic disease can further be divided as surgically resected cases and those receiving cranial RT alone. Nevertheless, we still believe that obtaining adequate preliminary data from this prospective pilot study is the most important mission now.

\section{Clinical implications and future directions}

First of all, whether this pilot study can be advanced to a larger-scale prospective study by introducing an appropriate control group deserves more discussion and debates. Conceptually thinking, patients fitting the same eligibility and randomized to receive conventional WBRT without hippocampal sparing should be the ideal control patient population. However, ethical considerations cannot be evaded at all, provided that HA-WBRT could achieve similar oncological outcomes and more favorable neurocognitive outcomes without violating the safety profile, as compared with conventional WBRT. ${ }^{[18,21]}$

Besides, for patients with a limited number of brain metastatic foci, particularly solitary brain metastasis, actually the current pattern of care preferred by some neurosurgeons/physicians is upfront stereotactic radiosurgery (SRS) but deferring the delivery of WBRT until intracranial failure; ${ }^{[40-43]}$ therefore, we can hypothesize that compared to initial SRS without upfront WBRT, the delivery of HA-WBRT would help patients with oligometastatic brain disease attain significantly better intracranial tumor control without compromising neurocognitive outcomes. As a re- sult, it justifies carrying out a prospective study comparing treatment effectiveness between HA-WBRT and upfront SRS alone for patients with oligometastatic brain disease.

\section{Conclusions}

In addition to intracranial disease control, common therapeutic and oncological outcomes, the integration of functional outcomes, including neurocognitive assessments, should provide further help for neuro-oncologists and health professionals when managing cancer patients harboring brain metastases. Furthermore, intr acranial control and functional preservation by reducing the dose delivered to the hippocampi during the WBRT course was generall y achieved in our prospective preliminary study, except for delayed verbal memory. Our study thus suggests that HA-WBRT should be a feasible and recommended technique which provides good intracranial control and preserves critical NCFs simultaneously in cancer patients harboring brain metastases with a favorable prognosis.

\section{Financial support and sponsorship}

Nil.

\section{Conflicts of interest}

There are no conflicts of interest.

\section{REFERENCES}

1. Norden AD, Wen PY, Kesari S. Brain metastases. Curr Opin Neurol 2005;18:654-61.

2. Koay E, Sulman EP. Management of Brain Metastasis: Past Lessons, Modern Management, and Future Considerations. Curr Oncol Rep 2012;14:70-8.

3. Maclean J, Fersht N, Singhera M, Mulholland P, Mckee O, Kitchen N, et al. Multi-disciplinary management for patients with oligometastases to the brain: Results of a 5 year cohort study. Radiat Oncol 2013;8:156.

4. Slotman B, Faivre-Finn C, Kramer G, Rankin E, Snee M, Hatton M, et al. Prophylactic cranial irradiation in extensive small-cell lung cancer. N Engl J Med 2007;357:664-72.

5. Aoyama H, Tago M, Kato N, Toyoda T, Kenjyo M, Hirota S, et al. Neurocognitive function of patients with brain metastasis who received either whole brain radiotherapy plus stereotactic radiosurgery or radiosurgery alone. Int J Radiat Oncol Biol Phys 2007;68:1388-95.

6. Kocher M, Soffietti R, Abacioglu U, Villa S, Fauchon F, Baumert BG, et al. Adjuvant Whole-Brain Radiotherapy Versus Observation After Radiosurgery or Surgical Resection of One to Three Cerebral Metastases: Results of the EORTC 22952-26001 Study. J Clin Oncol 2011;29:134-41.

7. Sun A, Bae K, Gore EM, Movsas B, Wong SJ, Meyers CA, et al. Phase III Trial of Prophylactic Cranial Irradiation Compared With Observation in Patients With Locally Advanced Non-Small-Cell Lung Cancer: Neurocognitive and Quality-of-Life Analysis. J Clin Oncol 2011;29:279-86. 
8. Welzel G, Fleckenstein K, Schaefer J, Hermann B, Kraus-Tiefenbacher U, Mai SK, et al. Memory Function before and after Whole Brain Radiotherapy in Patients with and without Brain Metastases. Int J Radiat Oncol Biol Phys 2008;72:1311-8.

9. Scoville WB, Milner B. Loss of recent memory after bilateral hippocampal lesions. J Neuropsychiatr Clin Neurosci 2000;12:103-13.

10. Mizumatsu S, Monje ML, Morhardt DR, Rola R, Palmer TD, Fike JR. Extreme sensitivity of adult neurogenesis to low doses of X-irradiation. Cancer Research 2003;63:4021-7.

11. Raber J, Rola R, Lefevour A, Morhardt D, Curley J, Mizumatsu S, et al. Radiation-induced cognitive impairments are associated with changes in indicators of hippocampal neurogenesis. Radiat Res 2004;162:39-47.

12. Rola R, Raber J, Rizk A, Otsuka S, Vandenberg SR, Morhardt DR, et al. Radiation-induced impairment of hippocampal neurogenesis is associated with cognitive deficits in young mice. Exp Neurol 2004; $188: 316-30$

13. Hellstrom NA, Bjork-Eriksson T, Blomgren K, Kuhn HG. Differential recovery of neural stem cells in the subventricular zone and dentate gyrus after ionizing radiation. Stem Cells 2009;27:634-41.

14. Lam LC, Leung SF, Chan YL. Progress of memory function after radiation therapy in patients with nasopharyngeal carcinoma. J Neuropsychiatr Clin Neurosci 2003;15:90-7.

15. Gondi V, Hermann BP, Mehta MP, Tome WA. Hippocampal Dosimetry Predicts Neurocognitive Function Impairment After Fractionated Stereotactic Radiotherapy for Benign or Low-Grade Adult Brain Tumors. Int J Radiat Oncol Biol Phys 2013;85:348-54.

16. Marsh JC, Ziel GE, Diaz AZ, Wendt JA, Gobole R, Turian JV. Integral dose delivered to normal brain with conventional intensity-modulated radiotherapy (IMRT) and helical tomotherapy IMRT during partial brain radiotherapy for high-grade gliomas with and without selective sparing of the hippocampus, limbic circuit and neural stem cell compartment. J Med Imaging Radiat Oncol 2013;57:378-83.

17. Ali AN, Ogunleye T, Hardy CW, Shu HK, Curran WJ, Crocker IR. Improved hippocampal dose with reduced margin radiotherapy for glioblastoma multiforme. Radiat Oncol 2014;9:20.

18. Gondi V, Mehta M, Pugh S, Tome W, Corn B, Caine C, et al. Memory Preservation with Conformal Avoidance of the Hippocampus during Whole-Brain Radiotherapy (WBRT) for Patients with Brain Metastases: Preliminary Results of RTOG 0933. Neuro Oncol 2013;15:94-5

19. Truc G, Martin E, Mirjolet C, Chamois J, Petitfils A, Crehange G. What place for the whole brain radiotherapy with hippocampal-sparing? Cancer Radiother 2013;17:419-23.

20. Ghia A, Tome WA, Thomas S, Cannon G, Khuntia D, Kuo JS, et al. Distribution of brain metastases in relation to the hippocampus: Implications for neurocognitive functional preservation. Int J Radiat Oncol Biol Phys 2007;68:971-7.

21. Gondi V, Pugh SL, Tome WA, Caine C, Corn B, Kanner A, et al. Preservation of memory with conformal avoidance of the hippocampal neural stem-cell compartment during whole-brain radiotherapy for brain metastases (RTOG 0933): A phase II multi-institutional trial. J Clin Oncol 2014;32:3810-6.

22. Wan JF, Zhang SJ, Wang L, Zhao KL. Implications for preserving neural stem cells in whole brain radiotherapy and prophylactic cranial irradiation: A review of 2270 metastases in 488 patients. J Radiat Res 2013;54:285-91.

Biomed J Vol. 38 No. 5

September - October 2015
23. Marsh JC, Herskovic AM, Gielda BT, Hughes FF, Hoeppner T, Turian J, et al. Intracranial metastatic disease spares the limbic circuit: A review of 697 metastatic lesions in 107 patients. Int J Radiat Oncol Biol Phys 2010;76:504-12.

24. Gaspar L, Scott C, Rotman M, Asbell S, Phillips T, Wasserman T, et al. Recursive partitioning analysis (RPA) of prognostic factors in three radiation therapy oncology group (RTOG) brain metastases trials. Int J Radiat Oncol Biol Phys 1997;37:745-51.

25. Gaspar LE, Scott C, Murray KCurran W. Validation of the RTOG recursive partitioning analysis (RPA) classification for brain metastases. Int J Radiat Oncol Biol Phys 2000;47:1001-6.

26. Han G, Liu D, Gan H, Denniston KA, Li SC, Tan WY, et al. Evaluation of the Dosimetric Feasibility of Hippocampal Sparing Intensity-Modulated Radiotherapy in Patients with Locally Advanced Nasopharyngeal Carcinoma. PLoS One 2014;9:e90007.

27. Wechsler D, Wechsler Memory Scale-Third Edition. Manual. 1997, San Antonio, TX: The Psychological Corporation.

28. Nelson HE. A modified card sorting test sensitive to frontal lobe defects. Cortex 1976;12:313-24

29. Wechsler D, Wechsler Adult Intellegence Scale ${ }^{\circledR}$-Third Edition. 1997, San Antonio, TX: The Psychological Corporation.

30. Gondi V, Tolakanahalli R, Mehta MP, Tewatia D, Rowley H, Kuo JS, et al. Hippocampal-Sparing Whole-Brain Radiotherapy: A "How-to" Technique Uing Helical Tomotherapy and Linear Accelerator-Based Intensity-Modurated Radiotherapy. Int J Radiat Oncol Biol Phys 2010;78:1244-52.

31. Atalar B, Modlin LA, Choi CY, Adler JR, Gibbs IC, Chang SD, et al. Risk of Leptomeningeal Disease in Patients Treated With Stereotactic Radiosurgery Targeting the Postoperative Resection Cavity for Brain Metastases. Int J Radiat Oncol Biol Phys 2013;87:713-8.

32. Harth S, Abo-Madyan Y, Zheng L, Siebenlist K, Herskind C, Wenz F, et al. Estimation of intracranial failure risk following hippocampal-sparing whole brain radiotherapy. Radiother Oncol 2013;109:152-8

33. Gondi V, Tome WA, Marsh J, Struck A, Ghia A, Turian JV, et al. Estimated risk of perihippocampal disease progression after hippocampal avoidance during whole-brain radiotherapy: Safety profile for RTOG 0933. Radiother Oncol 2010;95:327-31.

34. Barani IJ, Cuttino LW, Benedict SH, Todor D, Bump EA, Wu Y, et al. Neural stem cell-preserving external-beam radiotherapy of central nervous system malignancies. Int J Radiat Oncol Biol Phys 2007;68:978-85

35. Roman DD, Sperduto PW. Neuropsychological Effects of Cranial Radiation-Current Knowledge and Future-Directions. Int J Radiat Oncol Biol Phys 1995;31:983-98.

36. Gutierrez AN, Westerly DC, Tome WA, Jaradat HA, Mackie TR, Bentzen SM, et al. Whole brain radiotherapy with hippocampal avoidance and simultaneously integrated brain metastases boost: A planning study. Int J Radiat Oncol Biol Phys 2007;69:589-97.

37. Barani IJ, Benedict SH, Lin PS. Neural stem cells: Implications for the conventional radiotherapy of central nervous system malignancies. Int J Radiat Oncol Biol Phys 2007;68:324-33.

38. Barani IJ, Larson DA, Berger MS. Future directions in treatment of brain metastases. Surg Neurol Int 2013;4:S220-30.

39. Gondi V, Mehta MP. Novel insights into the management of brain metastases. Curr Opin Neurol 2010;23:556-62. 
40. Vogelbaum MA, Angelov L, Lee SY, Li L, Barnett GH, Suh JH. Local control of brain metastases by stereotactic radiosurgery in relation to dose to the tumor margin. J Neurosurg 2006;104:907-12.

41. Sheehan JP, Sun MH, Kondziolka D, Flickinger J, Lunsford LD. Radiosurgery for non-small cell lung carcinoma metastatic to the brain: Long-term outcomes and prognostic factors influencing patient survival time and local tumor control. J Neurosurg 2002;97:1276-81.

42. Sheehan JP, Sun MH, Kondziolka D, Flickinger J, Lunsford LD. Radiosurgery in patients with renal cell carcinoma metastasis to the brain: Long-term outcomes and prognostic factors influencing survival and local tumor control. J Neurosurg 2003;98:342-9.

43. Gans JH, Raper DM, Shah AH, Bregy A, Heros D, Lally BE, et al. The Role of Radiosurgery to the Tumor Bed After Resection of Brain Metastases. Neurosurgery 2013;72:317-25.

This is an open access article distributed under the terms of the Creative Commons Attribution-NonCommercial-ShareAlike 3.0 License, which allows others to remix, tweak, and build upon the work non-commercially, as long as the author is credited and the new creations are licensed under the identical terms.

For reprints contact: reprints@ medknow.com 